\title{
Erratum to: Ionospheric Effects of the Sudden Stratospheric Warming in 2009: Results of Simulation with the First Version of the EAGLE Model
}

\author{
M. V. Klimenko ${ }^{a, b}$, F. S. Bessarab ${ }^{a, b}$, T. V. Sukhodolov ${ }^{a, c, d}$, V. V. Klimenko ${ }^{a}$, \\ Yu. N. Koren'kov ${ }^{a,}$, I. E. Zakharenkova ${ }^{a}$, N. V. Chirik ${ }^{a, b}$, P. A. Vasil'ev ${ }^{a, b}$, \\ D. V. Kulyamin ${ }^{a, e}$, H. Schmidt $f$, B. Funke ${ }^{g}$, and E. V. Rozanov ${ }^{a, c, d}$ \\ ${ }^{a}$ Kaliningrad Branch of Pushkov Institute of Terrestrial Magnetism, Ionosphere and Radio Wave Propagation, \\ Russian Academy of Sciences, Kaliningrad, 236010 Russia \\ ${ }^{b}$ Immanuel Kant Baltic Federal University, Kaliningrad, 236041 Russia \\ ${ }^{c}$ Physikalisch-Meteorologisches Observatorium, World Radiation Center, Davos, Switzerland \\ ${ }^{d}$ Institute for Atmospheric and Climate Science, ETH Zurich, Zurich, Switzerland \\ ${ }^{e}$ Research Computing Center of the Moscow State University, Moscow, 119991 Russia \\ ${ }^{f}$ Max Planck Institute for Meteorology, Hamburg, Germany \\ ${ }^{g}$ Instituto de Astrofisica de Andalucia, CSIC, Granada, Spain \\ *e-mail: office@wdizmiran.ru \\ Received November 2, 2018; accepted November 2, 2018
}

DOI: $10.1134 / \mathrm{S} 199079311909001 X$

The author's name should read H. Schmidt instead of Kh. Shmidt

The original article can be found online at https://doi.org/10.1134/S1990793118040103. 